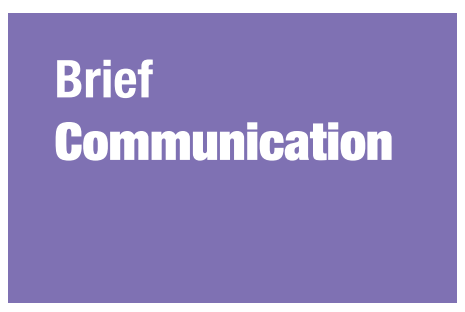

Submitted: 18 Sept 2016 Accepted: 20 Dec 2016 Online: 24 Feb 2017

\section{Limited Evaluation of Image Quality Produced by a Portable Head CT Scanner (CereTom) in a Neurosurgery Centre}

\author{
Ariz Chong Abdullah ${ }^{1,2,3}$, Johari Siregar Adnan ${ }^{1}$, Noor Azman A. \\ RAHMAN ${ }^{1}$, Ravikant PALUR ${ }^{1}$
}

1 Department of Neurosurgery, Hospital Sultanah Aminah, Jalan Persiaran Sultan Abu Bakar, 8010o Johor Bahru, Johor, Malaysia

2 Department of Neurosciences, School of Medical Sciences, Universiti Sains Malaysia, Jalan Sultanah Zainab 2, 16150 Kubang Kerian, Kelantan, Malaysia

3 Center for Neuroscience Services and Research, Universiti Sains Malaysia, Jalan Hospital USM, 16150 Kubang Kerian, Kelantan, Malaysia

To cite this article: Abdullah AC, Adnan JS, A Rahman NA, Palur R. Limited evaluation of image quality produced by a portable head CT scanner (CereTom) in a neurosurgery centre. Malays J Med Sci. 2017;24(1):104-112. https:// doi. org/10.21315/mjms2017.24.1.11

To link to this article: https://doi.org/10.21315/mjms2017.24.1.11

\title{
Abstract
}

Introduction: Computed tomography (CT) is the preferred diagnostic toolkit for head and brain imaging of head injury. A recent development is the invention of a portable CT scanner that can be beneficial from a clinical point of view.

Aim: To compare the quality of CT brain images produced by a fixed CT scanner and a portable CT scanner (CereTom).

Methods: This work was a single-centre retrospective study of CT brain images from 112 neurosurgical patients. Hounsfield units (HUs) of the images from CereTom were measured for air, water and bone. Three assessors independently evaluated the images from the fixed CT scanner and CereTom. Streak artefacts, visualisation of lesions and grey-white matter differentiation were evaluated at three different levels (centrum semiovale, basal ganglia and middle cerebellar peduncles). Each evaluation was scored 1 (poor), 2 (average) or 3 (good) and summed up to form an ordinal reading of 3 to 9.

Results: HUs for air, water and bone from CereTom were within the recommended value by the American College of Radiology (ACR). Streak artefact evaluation scores for the fixed CT scanner was 8.54 versus $7.46(Z=-5.67)$ for CereTom at the centrum semiovale, 8.38 $(\mathrm{SD}=$ 1.12) versus $7.32(S D=1.63)$ at the basal ganglia and $8.21(S D=1.30)$ versus $6.97(S D=2.77)$ at the middle cerebellar peduncles. Grey-white matter differentiation showed scores of $8.27(\mathrm{SD}=$ 1.04) versus 7.21 $(\mathrm{SD}=1.41)$ at the centrum semiovale, $8.26(\mathrm{SD}=1.07)$ versus $7.00(\mathrm{SD}=1.47)$ at the basal ganglia and $8.38(\mathrm{SD}=1.11)$ versus $6.74(\mathrm{SD}=1.55)$ at the middle cerebellar peduncles. Visualisation of lesions showed scores of 8.86 versus $8.21(Z=-4.24)$ at the centrum semiovale, 8.93 versus $8.18(Z=-5.32)$ at the basal ganglia and 8.79 versus $8.06(Z=-4.93)$ at the middle cerebellar peduncles. All results were significant with $P$-value $<0.01$.

Conchsions: Results of the study showed a significant difference in image quality produced by the fixed CT scanner and CereTom, with the latter being more inferior than the former. However, HUs of the images produced by CereTom do fulfil the recommendation of the ACR.

Keywords: CT, portable, image quality, CereTom, Hounsfield unit 


\section{Introduction}

Computed tomography (CT) scan is derived from the computer-processed combinations of many X-ray images to produce cross-sectional (tomographic) images of specific areas from a scanned object. Medical imaging is the most common application of CT scan for diagnostic and therapeutic purposes. CT scan has become the most accessible diagnostic toolkit for head and brain imaging.

The use of CT in general has led to a major shift since its invention in the 1970s and 1980s. The development of CT imaging with funding from the recording company EMI led to a Noble prize in Physiology or Medicine in 1979 (1). In emergency settings, CT scan is superior to magnetic resonance imaging (MRI). Indications for head CT imaging include head trauma, transient ischaemic attack, acute stroke and subarachnoid haemorrhage, among others.

The use of CT has increased greatly over the last two decades. An estimated 72 million CT scans were performed in the United States in 2007 (2). A recent advancement in CT imaging is the development of a mobile head CT scanner that can be beneficial from clinical and economical point of views.

Risks during transportation for imaging can be minimised if a mobile head CT scanner is available for patients in critical care. The risks include compromise of monitoring devices, intubation tubes, intravenous lines, hypotension, hypoxia and increased intracranial pressure. Even in a setting with a well-trained transport team comprised of senior staff, adverse events still occur $15 \%$ of the time (3).

Avoiding the transportation of patients who require imaging has other benefits as well. The amount of time required for imaging is reduced by eliminating the transport time for patients, and the utility of a fixed CT scanner of the hospital is improved by reducing the work load of the standard CT scanner (4). Therefore, the imaging for other non-critical care patients is facilitated, and their quality of care is improved.

The NeuroLogica CereTom CT scanner was introduced to the worldwide market in 2004. It is a portable CT scanner used primarily in neurological intensive care. It can also be used in the operating theatre to facilitate surgery or to verify surgical outcomes. CereTom can be used to replace the need for transporting a patient to a fixed CT scanner in the radiology department. A cost analysis conducted on the use of CereTom calculated a return on investment of $169 \%$, taking into consideration the cost of the machine (i.e., US\$359,000) and the single operator required to operate it $(4,5)$.

To date, only a few studies have been conducted to evaluate the image quality produced by a portable head CT scanner. Thus, we designed this study to evaluate the quality of CT brain images produced by a portable head CT scanner, CereTom, by comparing it with that of a fixed CT scanner in terms of the presence of streak artefacts, grey-white matter differentiation and visualisation of lesions. CT numbers (HUs) were measured for air, water and bone in the images produced by the portable CT scanner.

\section{Materials and Methods}

\section{Subjects}

This work was a single-centre retrospective study involving trauma and non-trauma patients admitted with intracranial pathology to the Neurosurgery Centre, Hospital Sultanah Aminah Johor Bharu (HSAJB), Malaysia, within four months from 1 December 2014 until 31 March 2015. The National Medical Research and Ethics Committee of the Ministry of Health, Malaysia, approved the study.

Patient registry of the admission record was screened together with the record book for portable CT scanner (CereTom) imaging in a duration of four months. Images from the same patient who had CT brain imaging performed from both CereTom and the fixed CT scanner within $48 \mathrm{~h}$ apart were selected. CT brain images harbouring intracranial pathology were taken from traumatic and non-traumatic patients of all age groups and sex. Images captured more than $48 \mathrm{~h}$ apart between the fixed CT scanner and CereTom or if any surgical intervention had been conducted between these imaging were excluded. Prominent artefacts from external devices preventing a clear assessment of CereTom images, fixed CT images or both were also excluded from this study. A final list of 112 pairs of imaging films of not more than $48 \mathrm{~h}$ apart were selected with no sampling conducted.

\section{Measures}

The CT numbers (HUs) of all the selected images from CereTom were measured for air, water and bone at the level of the middle cerebellar peduncles using the workstation of CereTom. The means of the measured values 
were compared with the CT number accuracy recommended by the ACR.

For each patient, the CT brain images conducted on both the fixed CT scanner and CereTom were evaluated independently by two neurosurgeons with five years' experience and one radiologist. All the images were evaluated for the presence of streak artefacts, grey-white matter differentiation and visualisation of lesions at the levels of the centrum semiovale, basal ganglia and middle cerebellar peduncles. Each evaluation was scored 1 for poor, 2 for average or 3 for good. The scores from the three assessors for every evaluation at different levels for both the fixed CT scanner and CereTom were summed up (range of score: $3^{-9}$ ) to obtain an ordinal reading.

These scores were evaluated for reliability using the Kappa test and showed to have a good agreement in the evaluation between the neurosurgeon and the radiologist.

Table 1. Reliability of image quality evaluation scores with fixed CT scanner

\begin{tabular}{cccccc} 
& \multicolumn{3}{c}{ Radiologist } & Total \\
Seore & $\mathbf{1}$ & $\mathbf{2}$ & $\mathbf{3}$ & $\mathrm{0}$ \\
& $\mathbf{1}$ & $\mathrm{O}$ & $\mathrm{O}$ & $\mathrm{0}$ & $\mathbf{1 8}$ \\
& 2 & $\mathrm{O}$ & 11 & 7 & 94 \\
& 3 & $\mathrm{O}$ & 2 & 92 & 112 \\
\hline
\end{tabular}

Kappa $=0.664$

$\mathrm{SE}$ of kappa $=0.103$

95\% confidence interval: From 0.463 to 0.866

Strength of agreement is considered to be 'good'

Table 2. Reliability of image quality evaluation scores with mobile CT scanner (CereTom)

\begin{tabular}{cccccc} 
& \multicolumn{3}{c}{ Radiologist } & \multicolumn{1}{c}{ Total } \\
Score & $\mathbf{1}$ & $\mathbf{2}$ & $\mathbf{3}$ & 3 \\
Neurosurgeon & $\mathbf{1}$ & $\mathrm{O}$ & 3 & $\mathrm{O}$ & 30 \\
& 2 & $\mathrm{O}$ & 21 & 9 & 79 \\
& 3 & $\mathrm{O}$ & 3 & 76 & 112 \\
\hline
\end{tabular}

Kappa $=0.665$

SE of kappa $=0.075$

95\% confidence interval: From 0.518 to 0.813

Strength of agreement is considered to be 'good' 
Brief Communication | Image quality of CereTom

\section{Statistical analysis}

All data collected were analysed using Statistical Package for Social Sciences (SPSS for Windows) version 21.0. Continuous variables, such as CT number (HU) accuracy, were expressed as mean with standard deviation (SD), and categorical variables were denoted as the number of subjects in percentage. As some of the data were normally distributed and others were not normally distributed, both parametric (paired $t$-test) and non-parametric (Wilcoxon signed rank test) tests were used, respectively, to test for statistical significance. Normally distributed data were presented as the mean with SD. Non-normally distributed data were presented as the median with 25th and 75th percentiles (interquartile range, IQR). A $P$-value of $<0.05$ was considered statistically significant.

\section{Results}

\section{Descriptive analysis}

A total of 224 CT brain images from 112 patients were retrieved in this study. Among the brain images, 112 were taken using the fixed
CT scanner and the other 112 were taken using CereTom. The images were obtained from 78 male patients $(69.6 \%)$ and 34 female patients (30.4\%) aged 13-64 years with a median age of 40.5 years $(I Q R=16.7)$. Among all cases, 78 were trauma (69.6\%) and 34 were non-trauma (30.4\%).

\section{CT number (HU)}

The mean CT number (HU) for accuracy was -972.65 ( $\mathrm{SD}=189.01$ ) for air, $1.40(\mathrm{SD}=$ 2.93) for water and 937.77 ( $\mathrm{SD}=60.58$ ) for bone. All the measured mean CT numbers (HU) for accuracy from CereTom were compared with the recommended value by the ACR, and all the mean values were within the recommended range.

\section{Streak artefacts, grey-white matter differentiation and visualisation of lesions}

For the streak artefacts, the image quality evaluation scores had a median of 8.54 for the fixed CT scanner versus 7.46 for CereTom ( $Z$ $=-5.67, P<0.01$ ) at the centrum semiovale, mean scores of $8.38(\mathrm{SD}=1.12)$ versus $7.32(\mathrm{SD}$ $=1.63)$ at the basal ganglia $(P<0.01)$ and 8.21

Table 3. Demographic characteristic of the study subjects $n=112$

\begin{tabular}{|c|c|c|c|}
\hline & & Frequency & Percentage (\%) \\
\hline \multirow[t]{2}{*}{ Gender } & Male & 78 & 69.6 \\
\hline & Female & 34 & 30.4 \\
\hline \multirow{2}{*}{ Classification of cases } & Trauma & 78 & 69.6 \\
\hline & Non Trauma & 34 & 30.4 \\
\hline
\end{tabular}

Table 4. CT number (Hounsfield unit, HU) accuracy for portable CT scanner (CereTom) and the recommended value by American College of Radiology (ACR)

\begin{tabular}{|c|c|c|c|}
\hline \multirow[b]{2}{*}{ Material } & \multicolumn{2}{|c|}{ Measured HU } & \multirow[b]{2}{*}{ Recommended HU } \\
\hline & Mean & $\begin{array}{c}\text { Standard deviation } \\
\text { (SD) }\end{array}$ & \\
\hline Air & -972.65 & 189.01 & Between -1005 and -970 \\
\hline Water & 1.40 & 2.93 & Between -7 and +7 \\
\hline Bone & 937.77 & 60.58 & Between 850 and 970 \\
\hline
\end{tabular}


$(\mathrm{SD}=1.30)$ versus $6.97(\mathrm{SD}=2.77)$ at the middle cerebellar peduncles $(P<0.01)$.

For the grey-white matter differentiation, the image quality evaluation scores had a mean of 8.27 (SD = 1.04) for the fixed CT scanner versus $7.21(\mathrm{SD}=1.41)$ for CereTom at the centrum semiovale, 8.26 (SD = 1.07) versus $7.00(\mathrm{SD}=1.47)$ at the basal ganglia and 8.38 $(\mathrm{SD}=1.11)$ versus $6.74(\mathrm{SD}=1.55)$ at the middle cerebellar peduncles. These results were significant with a $P$-value $<0.01$.

For the visualisation of lesions, the image quality evaluation scores had a median of 8.86 for the fixed CT scanner versus 8.21 for CereTom $(Z=-4.24, P<0.01)$ at the centrum semiovale, 8.93 versus 8.18 at the basal ganglia $(Z=-5.32$, $P<0.01)$ and 8.79 versus 8.06 at the middle cerebellar peduncles $(Z=-4.93, P<0.01)$.

Table 5. Image quality evaluation scores for the presence of streak artifacts, gray-white matter differentiation and visualization of lesions at the level of centrum semiovale between fixed CT scanner and portable CT scanner (CereTom)

\begin{tabular}{cccccc} 
& Levels & $\begin{array}{c}\text { Fixed CT scanner } \\
\text { Median (IQR) }\end{array}$ & $\begin{array}{c}\text { CereTom } \\
\text { Median (IQR) }\end{array}$ & Test statistics & P-value \\
Streak artifacts & $\mathrm{CS}$ & $8.54(0.24)$ & $7.46(1.16)$ & -5.67 & $P<0.001^{\mathrm{a}}$ \\
& $\mathrm{BG}^{*}$ & $8.38(1.12)$ & $7.32(1.63)$ & 6.72 & $P<0.001^{\mathrm{b}}$ \\
Gray-white matter & $\mathrm{MCP}^{*}$ & $8.21(1.30)$ & $6.97(2.77)$ & 6.98 & $P<0.001^{\mathrm{b}}$ \\
differentiation & $\mathrm{CS}^{*}$ & $8.27(1.04)$ & $7.21(1.41)$ & 7.48 & $P<0.001^{\mathrm{b}}$ \\
Visualisation of & $\mathrm{BG}^{*}$ & $8.26(1.07)$ & $7.00(1.47)$ & 7.88 & $P<0.001^{\mathrm{b}}$ \\
lesions & $\mathrm{MCP}^{*}$ & $8.38(1.11)$ & $6.74(1.55)$ & 10.12 & $P<0.001^{\mathrm{b}}$ \\
& $\mathrm{CS}$ & $8.86(0.09)$ & $8.21(0.34)$ & -4.24 & $P<0.001^{\mathrm{a}}$ \\
& $\mathrm{BG}$ & $8.93(0)$ & $8.18(0.57)$ & -5.32 & $P<0.001^{\mathrm{a}}$ \\
\hline
\end{tabular}

$\mathrm{CS}=$ Centrum semiovale $; \mathrm{BG}=$ Basal ganglia $; \mathrm{MCP}=$ Middle cerebellar peduncle

$\mathrm{IQR}=$ Interquartile range

${ }^{*}$ Mean (Standard deviation)

${ }^{a}$ Wilcoxon Signed Ranks test (data were not normally distributed)

${ }^{\mathrm{b}}$ Paired $t$-test (data were normally distributed)

\section{Discussion}

In our practice at a tertiary care hospital with an active neurological intensive care unit, critically ill patients commonly undergo multiple cranial CT examinations over days to weeks. As these examinations are clinically indicated and required for appropriate patient care, a reduction in image quality below that required for minimal diagnostic accuracy would clearly be counterproductive.

This study revealed that CT brain imaging was conducted more commonly in male than in female patients with a ratio of 2.3 male to 1 female. The age group of the patients in this study ranged from 13 to 64 years with a median age of 40.5 years $(I Q R=16.7)$. Majority of the cases were trauma patients at $69.6 \%$, and the non-trauma cases accounted for $30.4 \%$. The higher male preponderance and trauma cases reflected the higher involvement of males than of females in road traffic accidents in Malaysia.

In this study, the measured air, water and bone HUs from CereTom were within the range recommended by the ACR. Our results showed that the image quality evaluation scores for the presence of streak artefacts, grey-white matter differentiation and visualisation of lesions for CereTom were significantly lower than those for the standard fixed CT scanner. Significant differences were found in the image quality evaluation scores at all three tested levels.

The CT numbers (HU) for accuracy for air, water and bone measured from CereTom were within the recommended range by the ACR. The HU is a quantity commonly used in CT scanning to express CT numbers in standardised and convenient forms. The $\mathrm{HU}$ 
scale is a linear transformation of the original linear attenuation coefficient measurement into one in which the radiodensity of distilled water at standard pressure and temperature (STP) is defined as o HU, and the radiodensity of air at STP is defined as -1000 HU. The CT number values are clinically relevant in determining the composition of various tissues in the body. Accuracy is important in the characterisation of tumours, intracranial haemorrhage and foreign bodies, among others.

In our study, the image quality evaluation scores for the presence of streak artefacts from images at all three levels produced from CereTom were significantly lower than those from images produced from the fixed CT scanner. The difference was more apparent at the middle cerebellar peduncle level. Streak artefacts in the skull base could degrade image quality to the non-diagnostic level. Streak artefacts were produced when the object moved, under sampled or corrupted because of data sampling errors (6). In our study, all three assessors considered the artefact to be mainly caused by the partial volume effect and movement.

Generally, images produced by a CT scanner are accurate representations of the scanned object. However, artefacts are commonly encountered and may obscure intracranial pathology. CT artefacts have many causes, including noise, beam hardening, scatter, pseudoenhancement, motion, cone beam, helical, ring and metal artefacts. The evaluation of streak artefacts with CereTom was noted to have comparable results with standard CT scanners (7).

The brain consists of grey and white matter structures differentiated by density in CT. White matter has a high content of myelinated axons. Grey matter contains relatively few axons and a high number of cell bodies. As myelin is a fatty substance, it has relatively lower density than the cellular grey matter. Therefore, white matter appears blacker than grey matter. Our study showed that the grey-white matter differentiation was significantly better in the images from the fixed CT scanner than in those from CereTom. The difference in observation was considered to be caused by patient movement, partial volume effect and possible small cavity within the posterior fossa, which all make comparison difficult.

Various methods of quantification, including measurement of $\mathrm{HU}$, subtracting the values of grey and white matter from the cerebrospinal fluid or measuring both against a skull water phantom $(8,9,10)$, have been used to differentiate between grey and white matter. Generally, a decreased distinction between grey and white matter in CT predicts a poor outcome after cerebral insult. We hypothesised that this predictive capacity could be increased if the analysis was more quantitative.

In general, both the fixed CT scanner and CereTom obtained good image quality evaluation scores for the visualisation of lesions. However, the evaluation scores of the fixed CT scanner were significantly better than those of CereTom. The CT scanner has the advantage of a prompt visualisation of acute intracranial pathology with their location sites, mass effect, oedema and identification of size and configuration of the ventricular system and subarachnoid spaces, bone fractures, or presence of foreign bodies. Our three assessors preferred the fixed CT scanner to CereTom in the visualisation of lesions. This assessment is partly due to the fact that scale for measurement was not provided in the CereTom images and to the inappropriate gantry of imaging, thus making judgment more difficult.

\section{Clinical Relevance}

The published literature on portable CT scanners is limited and mainly covers two topics: (1) image quality and dose (compared with a fixed CT scanner) and (2) patient welfare versus economic advantages and disadvantages (bringing the CT scanner to the patient rather than the patient to the scanner).

A portable CT scanner is designed for specific clinical circumstances in which the usage of a standard fixed CT scanner or other existing imaging modality is unfeasible or difficult to obtain (11). The greatest strength of CereTom is its mobility.

The major advantage of CereTom is its ability to be transported and used at the patient's bedside unlike a fixed CT scanner that requires the patient to be transported to the imaging department. This equipment is particularly important in cases in which the patient is too ill or unstable to transport $(12,13,14)$. The need for staff from the critical care unit to accompany the patient to the imaging department is also minimised $(15,16)$.

The relatively small and compact size and mobility of CereTom enable it to move freely in and out of the operating theatre. It does not require specialised equipment and can easily fit 
in existing operating theatres. It has scanning capabilities that enable contrast study. Although radiation exposure is possible, the actual risk is quite low.

The risk of intraoperative CT is considered small compared with the potential risks of damaging neural elements, leaving residual tumour or having surgical complications, such as postoperative bleeding, morbidity and cost of repeat surgery, to the patient. An analysis by intraoperative guidance with MRI showed a 55\% reduction of length of stay, lower repeat surgery rates and lower total overall hospital cost by $46 \%$ $(16,17)$. Intraoperative CT may possibly produce the same results.

CereTom is a potential alternative for diagnosing brain death for critically ill or unstable patients. CT perfusion scans can assess the extent of infarct penumbra and indicate the possibility of early thrombolysis in cases of ischaemic stroke (19).

The operation of CereTom can be performed by one radiographer (20). Ease of use and operation of the system is considered good and can improve with familiarity. The ease of patient positioning is dependent on the patient's body size and number of attachments. Positioning is easy as long as a scan board is used.

Although imaging the patient at bedside has advantages, including decreased risk of adverse effects during transportation especially for a critically ill patient, the relative cost, image quality, diagnostic benefit and radiation dose must also be considered.

The height and weight of the scanner can make moving the unit difficult for certain operators, especially when travelling over long distances. Movement is also difficult over uneven floor surfaces, cushioned or carpeted flooring (19). The difficulty in moving the portable CT scanner (CereTom) is especially apparent when the patient and the machine are situated at different levels of the same building or in different buildings. Having two people move the system is advisable.

As there is no means to tilt the gantry of the scanner, the positioning of the patient can be difficult at times, and irradiating the orbits during brain scanning procedures is possible (18). However, the potential risk is outweighed when considering the clinical benefits from the scan. Therefore, justification must be made in terms of the overall exposure and that of the eyes.

The structure of our neurosurgery ward in HSAJB, including the intensive care unit, makes the mobilisation of a portable CT scanner to the patient for imaging difficult. Therefore, a designated area $50 \mathrm{~m}$ from the ward with the highest possible usage, which is our neurosurgery intensive care unit, has been set up to place the CereTom. With this setup, the CereTom can be easily moved to the patient's bed for scanning, and the distance is significantly short. The total duration for imaging is remarkably reduced, and faster decisions for intervention can be made.

\section{Conclusion}

In conclusion, a difference is observed in terms of image quality between the images produced by a fixed CT scanner and those by a portable CT scanner (CereTom), with the latter being inferior. However, the $\mathrm{HU}$ of the images produced by CereTom do fulfil the recommendations by the ACR.

\section{Acknowledgements}

I would like to express my utmost gratitude to all those who have helped me directly or indirectly, and supported me in doing this research study and to complete this thesis. I would like to thank my family, especially my beloved parents for their love, care and immeasurable support throughout the duration of my rigorous study in Universiti Sains Malaysia. My special thanks to my ever understanding wife, Adlena for her thoughtfulness and patience. They are my source of motivation to strive hard to contribute to the society through research and clinical work in the field of neurosurgery.

I am most honoured to express my sincere appreciation to my supervisor, Datuk Dr. Johari Siregar Adnan, Consultant Neurosurgeon of Hospital Sultanah Aminah Johor Bahru, for providing me guidance, suggestions as well as encouragement and advice all the time during the research and writing of this thesis. I would like to extend my appreciation to Dr. Azman, Dr. Ravi Palur, Dr. Sharon and Dr. Khatijah for their assistance, constructive critisms, patience and suggestions in helping me with this study.

I am indebted to Professor Dato Dr. Jafri Malin Abdullah, the head of Department of Neurosciences, School of Medical Sciences, Universiti Sains Malaysia for his motivation and support in conduction of this study. Not forgetting, my heartfelt thanks to all the 
Brief Communication | Image quality of CereTom

lecturers, Associate Professor Dr. Zamzuri Idris, Dr. Badrisyah and Dr. Regunath, for valuable suggestion.

Not to forget, my sincere appreciation to all my fellow colleagues, ward staffs, operating theatre staffs and all my friends who have brought me cheers and supports.

\section{Authors' Contributions}

Conception and design: ACA, RP, NAAR, JSA Analysis and interpretation of the data: ACA, RP Drafting of the article: ACA, RP

Critical revision of the article for important intellectual content: NAAR, JSA

Final approval of the article: JSA

Provision of study materials or patients: ACA, NAAR

Statistical expertise: ACA

Obtaining of funding: ACA

Administrative, technical or logistic support: ACA, NAAR

\section{Correspondence}

Dr Ariz Chong Abdullah

Neurosurgeon

MBBS, International Medical University

Master of Surgery (Neurosurgery), Universiti Sains

Malaysia

Department of Neurosurgery,

Hospital Sultanah Aminah,

Jalan Persiaran Sultan Abu Bakar,

80100 Johor Bahru,

Johor, Malaysia

Tel: +6012 7916352

E-mail: arizchong@hotmail.my

\section{References}

1. Alexander RE, Gunderman RB. EMI and the first CT scanner. J Am Coll Radiol. 2010;7(10):778781. https://doi.org/10.1016/j.jacr.2010.06.003

2. Berrington G, Mahesh M, Kim KP, Bhargavan M, Lewis R, Mettler F, et al. Projected cancer risks from computed tomographic scans performed in the United States in 2007. Arch Intern Med. 2009;169(22):2071-2077. https://doi. org/10.1001/archinternmed.2009.440

3. Waydhas C. Intrahospital transport of critically ill patients. Crit Care Med. 1999;3:83-89.
4. Masaryk T, Kolonick R, Painter T, Weinreb DB. The economic and clinical benefits of portable head/neck CT imaging in the intensive care unit. Radiol Manage. 2008;30:50-54.

5. Mayo-Smith WW, Rhea JT, Smith WJ, Cobb CM, Gareen IF, Dorfman GS. Transportable versus fixed platform CT scanners: comparison of costs. Radiology. 2003;226:63-68. https://doi. org/10.1148/radiol.2261012047

6. Robert P, Kumar S, Lowell H, Keith AK. CT artifact recognition for the nuclear technologist. $J$ Nucl Med Technol. 2008;36:79-81. https://doi. org/10.2967/jnmt.107.047431

7. Rumboldt Z, Huda W, All JW. Review of portable CT with assessment of a dedicated head CT scanner. AJNR Am $J$ Neuroradiol. 2009;30(9):1630-1636. https://doi.org/10.3174/ ajnr.A1603

8. Hounsfield GN. Historical notes on computerized axial tomography. $J$ Can Assoc Radiol. 1976;27:135-142.

9. Avrahami E, Katz R, Rabin A, Friedman V. CT diagnosis of non-traumatic subarachnoid haemorrhage in patients with brain edema. Eur $J$ Radiol. 1998;28:222-225. https://doi. org/10.1016/So720-048X(97)00162-9

10. Boris P, Bundgaard F, Olsen A. The CT Hounsfield unit number of brain tissue in healthy infants: a new reliable method for detection of possible degenerative disease. Childs Nerv Syst. 1987;3:175-177. https://doi.org/10.1007/ BFoo717896

11. Maher MM, Hahn PF, Gervais DA, Seoighe B, Ravenscroft JB, Mueller PR. Portable abdominal CT: analysis of quality and clinical impact in more than 100 consecutive cases. AJR Am J Roentgenol. 2004;183:663-670. https://doi. org/10.2214/ajr.183.3.1830663

12. McCunn M, Mirvis S, Reynolds N, Cottingham C. Physician utilization of a portable computed tomography scanner in the intensive care unit. Crit Care Med. 2000;28:3809-3813. https://doi. org/10.1097/00003246-200012000-00008

13. Gunnarsson T, Theodorsson A, Karlsson P, Fridriksson S, Bostrom S, Persliden J, et al. Mobile computerized tomography scanning in the neurosurgery intensive care unit: increase in patient safety and reduction of staff workload. $J$ Neurosurgery. 2000;93:432-436. https://doi. org/10.3171/jns.2000.93.3.0432 
14. Teichgraber UK, Pinkernelle J, Jurgensen JS, Ricke J, Kaisers U. Portable computed tomography performed on the intensive care unit. Intensive Care Med. 2003;29:491-495. https:// doi.org/10.1007/so0134-002-1606-x

15. Peace K, Wilensky EM, Frangos S, MacMurtrie E, Shields E, Hujcs M, et al. The use of a portable head CT scanner in the intensive care unit. $J$ Neurosci Nurs. 2010;42(2):109-116. https://doi. org/10.1097/JNN.obo13e3181ce5c5b

16. Stevens GC, Rowles NP, Foy RT, Loader R, Barua N, Williams A, et al. The use of mobile computed tomography in intensive care: regulatory compliance and radiation protection. $J$ Radiol Prot. 2009;29:483-490. https://doi. org/10.1088/0952-4746/29/4/002
17. Hall WA, Kowalik K, Liu H, Truwit CL, Kucharezyk J. Costs and benefits of intraoperative MR-guided brain tumor resection. Acta Neurochir Suppl. 2003;85:137-142. https://doi. org/10.1007/978-3-7091-6043-5_19

18. Hall WA, Truwit CL. Intraoperative MRguided neurosurgery. J Magn Reson Imaging. 2008;27(2):368-375. https://doi.org/10.1002/ jmri.21273

19. CEP evaluation report 09039: NeuroLogica CereTom portable CT scanner. NHS Purchasing and Supply Agency; 2009.

20. NeuroLogica Corporation. NL 3000 CereTom user manual; 2007. 